\title{
Effect on Microstructure and Nanoindentation of a AlCoFeMoNi High Entropy Alloy.
}

\author{
F.J. Baldenebro-Lopez ${ }^{1}$, C.D. Gómez-Esparza ${ }^{2}$, H. Camacho-Montes ${ }^{1}$ and R. Martínez-Sánchez ${ }^{3}$ \\ 1. FIM-Universidad Autónoma de Sinaloa, Los Mochis, Sinaloa, México. \\ 2. Centro de Investigación en Materiales Avanzados (CIMAV), Chihuahua, Chih., México. \\ 3. IIT-Universidad Autónoma de Ciudad Juárez., Ciudad Juárez, Chih., México.
}

High entropy alloys (HEAs) have been known as a new type of material with unusual properties. HEAs contain at least five major elements in equiatomic or near-equiatomic proportions [1], and in general tend to form simple FCC and/or BCC microstructures in form of solid solutions (SS) [2]. Several chemical systems in HEAs have been investigated, but Mo-Ti couple has been rarely reported. The aim of this work was to characterize the microstructure of HEA produced by mechanical alloying (MA) and arc melting on AlCoFeMoNi alloy.

The HEAs have been fabricated by different routes. The melting process has become the preferred technique to produce these alloys. It is well known that samples produced by MA followed by consolidation, possess a higher pore density than samples fabricated by casting. However, the melting route leads to the segregation problem, while in the MA process, homogenous chemical distribution and solid solubility extension can be reached. However, in the field of HEAs, the synthesis of new alloys attempts to solve the limitations of traditional metallurgy techniques.

In this work, elemental powders supplied by Alfa Aesar with purity higher than $99.5 \%$ in weight $\mathrm{Al}, \mathrm{Co}$, $\mathrm{Fe}$, Mo and Ni were initially weighed and mixed. The MA was performed in a high-energy ball mill (SPEX-8000M) for $10 \mathrm{~h}$ periods, under an argon atmosphere. Methanol was used as a process control agent to avid metal agglomeration. The milled powders were melting by electric arc furnacean argon atmosphere to prevent oxidation. The ingots were remelted at least 5 times to improve chemical homogeneity.

In the Figure 1 a TEM micrograph of a single powder particle after milling is presented, showing only one phase in powders after $10 \mathrm{~h}$ of milling. Figure 2 shows SEM images of a representative microstructure after arc melting. The sample exhibit the formation of a gray phase (Al-Co-Ni), a bright phase (Fe-Mo-Co), and discontinuous bright phase (Mo). Figure 3 shows the size of the nanoindentations in each phase, being able to observe that the phase rich in molybdenum is the one with the highest hardness. Figure 4 shows the distribution of the alloying elements in each of the present phases.

A nanocrystalline AlCoFeMoNi alloy was successfull synthesized by mechanical alloying. Consolidated samples by arc melting presented microstructure with three phases according to electron microscopy observations.

References:

[1] Y.L. Chen, et al, Alloys Compd. 477 (2008), p. 696.

[2] T.T. Shun and Y.C. Du, J. Alloys Compd. 479 (2009), p. 157. 


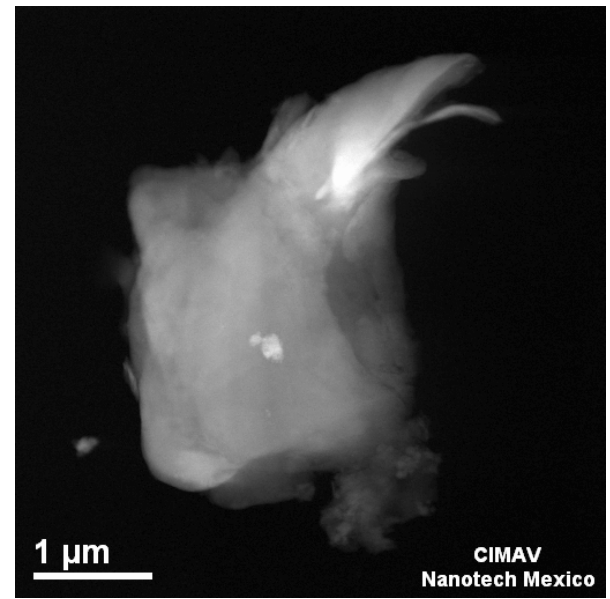

Figure 1. TEM micrographs of AlCoFeMoNi powder alloy after $10 \mathrm{~h}$ of milling.

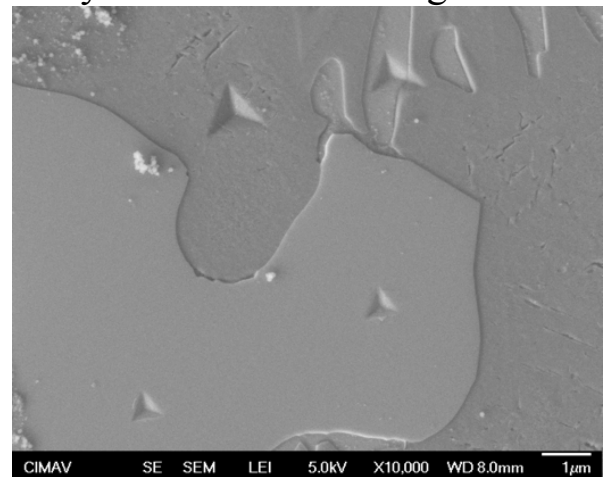

Figure 3. SEM images of nanoindented phases.
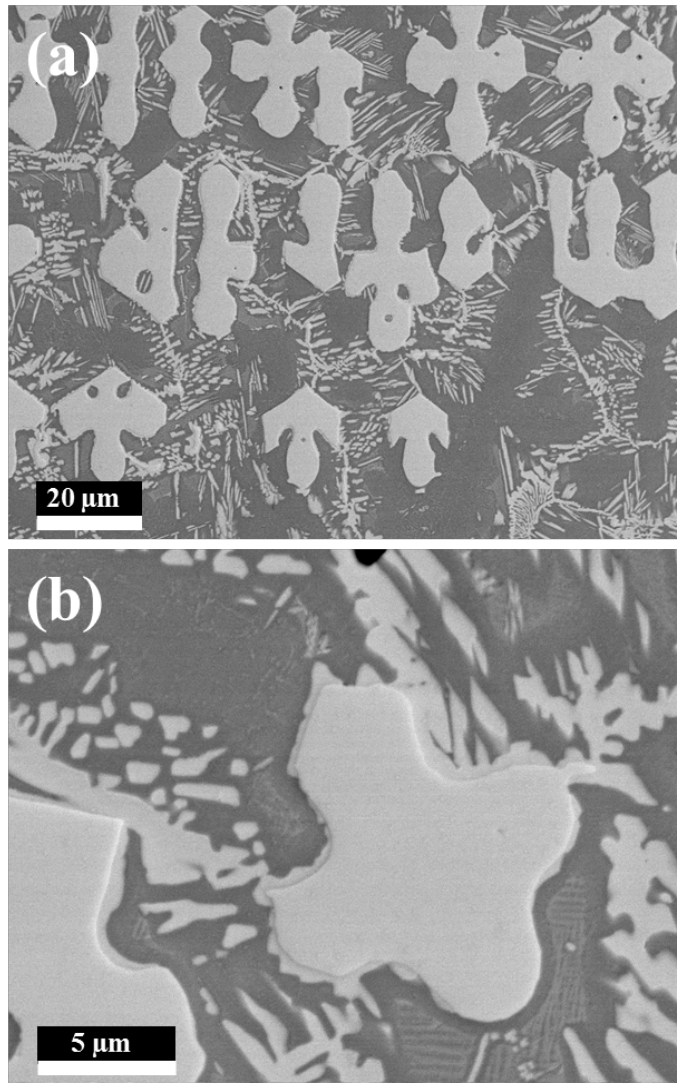

Figure 2. SEM images of samples: (a) $1000 \mathrm{X}$ and (b) 5000X.
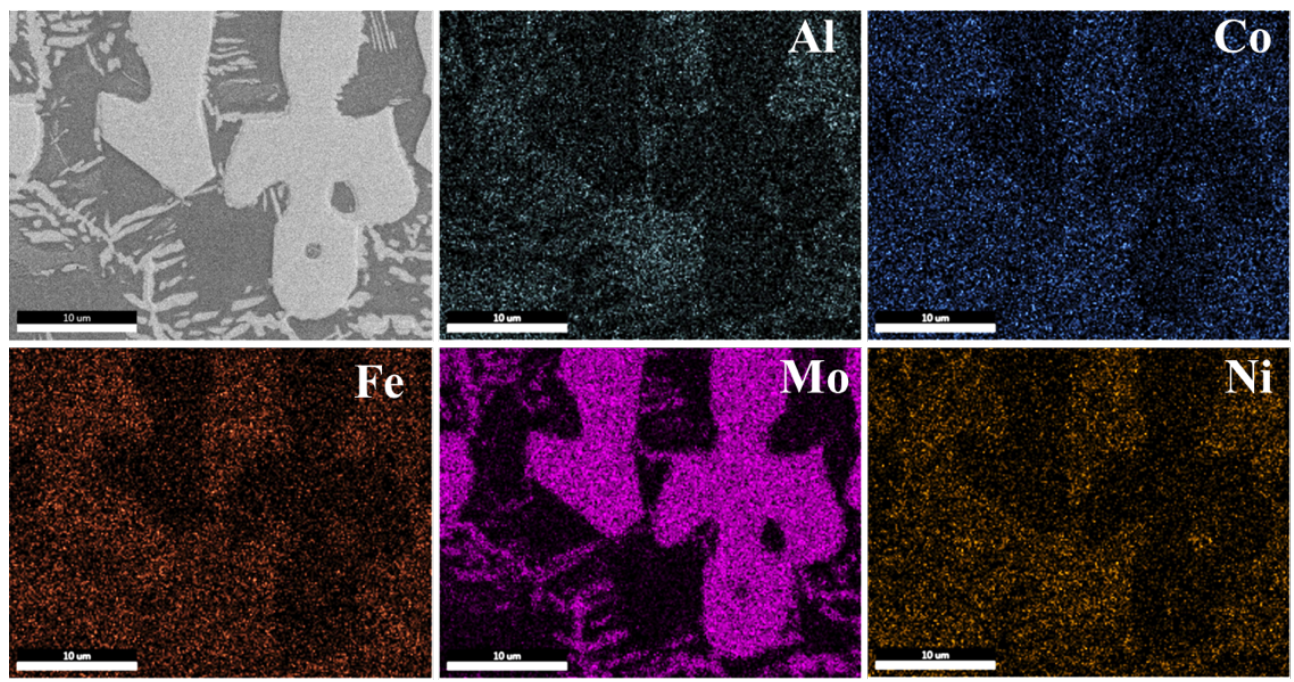

Figure 4. SEM-EDS mapping of the phases. 